\title{
The Galactic Ecosystem
}

\author{
Michael Burton \\ University of New South Wales, School of Physics, Sydney, NSW 2052, \\ Australia
}

\begin{abstract}
We discuss how the process of star formation across the spiral arms of galaxies functions as an ecosystem. The carbon abundance plays a crucial role in both its self-regulation and evolution.
\end{abstract}

\section{Star Formation across the Spiral Arms}

Seen face-on, the visible light from the Galaxy appears as a spiral. While only forming a small fraction of the Galaxy, the spiral arms are its most luminous part, as it is where star formation takes place. They are a "compression wave". Gas flows into them, is compressed, and a portion emerges as new stars. The rate of star formation, and its efficiency, are controlled by feedback processes. It is driven by the exchanges of matter, and the flow of energy, between the stars and the interstellar medium (ISM). It also acts in an autonomous manner, yet is able to self-regulate the rate and content of star formation. These are the processes that are evident in ecosystems-systems where energy flows and material cycles are maintained in an apparently stable, but non-equilibrium, state, by a process of self-regulation. This paper discusses how the concept of an ecosystem can be used to provide insight into understanding the evolution of the Galaxy. It has been stimulated by the discussion in Smolin (1997).

Stars form from the collapse of clouds of molecular gas under the influence of gravity. The compression wave of an approaching spiral arm can provide a triggering mechanism to overcome the support mechanisms which hold a cloud up against gravity. Once star formation starts in a cloud it is then dominated by the most massive stars which form there. While these stars have masses a few times the Sun's $\left(10-100 \mathrm{M}_{\odot}\right)$, their luminosities range from $10^{3}-10^{6} \mathrm{~L}_{\odot}$, vastly greater than it. They create ionized bubbles, which rapidly expand to envelope and disrupt the surrounding molecular cloud. The spiral arms are thus lit up by the massive stars forming in them. Yet they only make up a tiny percentage of the number of stars that are forming at any given time, or indeed of the total number of stars in the Galaxy. The rate of star formation in a particular cloud depends on the local environment of the cloud, and on the stars already forming in it. Massive stars not only emit prodigious amounts of ionizing radiation, but they also eject tremendous winds. These drive shock waves through the surrounding molecular clouds. Such interactions can serve to cause further star formation, compressing the gas and triggering collapse. Or they can hinder it, breaking up the molecular cloud and providing turbulent support against further collapse. Star formation is thus self-regulated by the most massive stars which form as a part of the process. 


\section{Carbon Catalysis}

The rate of star formation, and the mass of the stars that form, is also strongly influenced by the chemical composition of the clouds. To collapse efficiently a cloud must be able to quickly release its gravitational energy. Otherwise it will heat up, generating thermal pressure to support the cloud against further collapse. The energy is released through emission lines from atoms and molecules present in the cloud. Below $\sim 1000 \mathrm{~K}$ the dominant element, hydrogen, does not provide an efficient coolant in either its atomic or molecular form. The efficiency of cooling is determined by the elemental abundance of the cloud, particularly the amount of carbon present. In either its singly ionized form emitting at a wavelength of $158 \mu \mathrm{m}$, in molecular form emitting several lines in the millimetre portion of the spectrum, or as part of large carbon-ring molecules (polycyclic aromatic hydrocarbons, or PAHs), carbon provides the dominant cooling mechanism across a wide range of density conditions prevalent in molecular clouds (e.g., Tielens \& Hollenbach 1985). The abundance of carbon in the Galaxy has been increasing as the Galaxy has aged. Thus its influence in regulating the rate of star formation, the resultant distribution of stellar masses, and even the processes taking place during the lives of individual stars, has changed over the history of the Galaxy. Carbon is produced by nucleosynthesis within the cores of stars. It is cycled into the ISM by the massive stars, either in radiation-driven stellar winds having been dredged up from their cores (e.g., Maeder 1992), or in the ejecta of supernova explosions at the end of the star's life.

\section{The Galactic Ecosystem}

The stars themselves are far from thermal equilibrium, with the energy flows from their surface's radiating into cold space. The stars are stable, but nonequilibrium systems, that have their own cycle of birth, life and death. The timescale for the Galactic ecology is determined by the rate of star formation and the lifetime of the most massive stars (a few million years). This ecology must have existed over the life of the Galaxy. It is driven by the energy flows from the massive stars, and the material cycle through these same stars. Carbon, and heavier elements, are created in the massive stars, and released through winds and supernova explosions. They cycle between the various phases of the interstellar medium, before again being incorporated into stellar systems. Further star formation in a molecular cloud is self-regulated by the massive stars already forming there, and by the cooling agents present. These agents gradually change as the elemental abundances, particularly that of carbon, increase, on average, as the Galaxy ages ${ }^{1}$. A feedback process operates which both regulates the star formation rate and determines the stellar initial mass spectrum.

The elements of an ecosystem are therefore in operation in the Galaxy; energy flows and materials cycles, driven by the massive stars, in systems that are far from thermal equilibrium, and whose end result is determined by a process of self-regulation. In this picture the interstellar medium serves as the atmosphere

\footnotetext{
${ }^{1}$ This does not mean that the abundance increases in a uniform or monotonic fashion; the variation in abundances across the Galaxy is a measure of the diversity of the system.
} 
of the Galaxy, the medium for its evolution. Through it energy flows, and gas is cycled, from one form to another. Stars form the reservoirs and the interstellar medium the exchange pools for the galactic ecosystem.

Despite the delicate balance and interplay between many aspects of the star formation cycle, the system has continued to operate for over ten billion years. It needs to be asked whether this system is auto-catalytic, returning to its starting point, or whether it is evolving in time, with new features appearing as the Galaxy ages? Such behaviour is a necessary consequence of the operation of an ecosystem, and provides a means of examining the merits of this model. We argue below that the Galaxy displays a weak form of evolution, evident through the change in the initial mass function with time, and therefore in the type of stars which form in it.

\section{The Evolution of the Initial Mass Function?}

Star formation produces a range of stellar masses, with the initial mass function (IMF) describing how many stars of a particular mass form. The life story of an individual star is determined largely by its mass, and is very different for low mass and high mass stars. The average value of the IMF can be measured and is heavily weighted towards lower mass stars. The abundance of elements within a cloud, particularly that of carbon, affects the IMF due to their role in the cooling of a collapsing cloud.

At early times in the Galaxy the IMF would have been weighted towards higher mass stars than it is today, impeding the collapse at low masses (Larson 1998). This is because at lower elemental abundances molecular clouds are, on average, hotter than today. Higher masses were thus needed at early epochs to overcome the thermal pressures for collapse to occur. As the Galaxy has aged, and the carbon abundance increased, the IMF has changed, though the manner in which it has done so is not well understood.

Thus the Galaxy is displaying a very simple form of evolution, through the change in the IMF as it ages, and therefore in the community of stars that exist within it. New types of stars (i.e. with higher "metallicities") appear as the Galaxy evolves. This occurs due to a limited form of information passage from one generation of stars to the next, through the changing distribution of the initial elemental abundances. The stars that form in one generation influence the type and number of stars that form in the next.

There is thus self-replication (i.e. more stars are created) with variation (i.e. a changing IMF and stellar behavioural properties, as the elemental abundances build up in successive generations), and these continue to replicate with variation, at least until all the raw material to form stars has been exhausted. This is the basic process associated with evolution (e.g., Ehrlich 1991).

\section{Examining the Case for Carbon Self-Regulation}

Can this description of the Galactic ecosystem help us to understand particular phenomena that take place within it? A test of its merit would be if it helps predict behaviour in certain circumstances. The suggestion that star formation has been self-regulated over the ten billion years of galactic history is most open to 
criticism. We have hypothesised that it is the massive stars, and the role of the heavy elements, particularly carbon, that is central to this process. This can be subject to testing, as the origin of the elements, and their distribution through the Galaxy, is not yet well understood. Currently the evidence does indeed favour the production of carbon through winds from massive $\left(>10 \mathrm{M}_{\odot}\right)$ stars as being the dominant contributor (e.g., Henry, Edmunds \& Koppen 2000). It provides an effectively instantaneous production of carbon compared to galactic timescales, as it does not even require the star to complete its life before enriching the gas. As the carbon abundance increases, then so will the regulatory mechanism alter.

Carbon can also be produced, however, via the triple-alpha reaction, by all stars greater in mass than Solar. The lower mass stars can supply carbon into the interstellar medium via mass loss during the planetary nebula phase at the end of their red giant stage. Since low mass stars greatly outnumber high mass ones, this could provide the dominant method of carbon-enrichment (e.g., Gustafsson et al 1999). If so, then the means of self-regulation of the system described here, which is driven by the massive stars, would fail. An ecosystem would not then operate.

There have been shifting views with regards to the relative roles of high and low mass stars in the enrichment process. Mass loss rates are sensitive to the carbon abundance in stars, being greater at higher abundance due to the increased opacity in their surface layers, improving the efficiency of the radiative driving mechanism of winds. On the other hand, as the Galaxy ages, and the number of (long-lived) low mass stars increases, the relative importance of the lower mass stars will increase, possibly removing the self-regulatory mechanism of carbon production from the massive stars (e.g., Liang, Zhao \& Shi 2001).

\section{Conclusions}

We have argued that the star formation cycle, through the spiral arms of the Galaxy, operates as an ecosystem. Our Galaxy, and indeed spiral galaxies in general, must be the first ecosystems to form within the Universe, within the first 3 billion years of its existence. They are also likely to be the simplest such systems to exist within it, with their operation determined entirely by physical processes. Carbon plays a central role in both the self-regulation of star formation and in the evolution of the Galaxy.

\section{References}

Ehrlich, P. 1991, Scientists on Gaia, ed. S. Schneider \& P. Boston, MIT, 19 Gustafsson, B. et al. 1999, A\&A, 342, 426

Henry, R. B. C., Edmunds, M. G., \& Koppen, J. 2000, ApJ, 541, 660

Larson, R. B. 1998, MNRAS, 301, 569

Liang, Y. C., Zhao, G., \& Shi, J. R. 2001, A\&A, 374, 936

Maeder, A. 1992, A\&A, 264, 105

Smolin, L. 1997, The Life of the Cosmos, (London: Phoenix)

Tielens, A. G. G. M., \& Hollenbach, D. J. 1985, ApJ, 291, 722 